Elemente.

Oskulationsepoche 1907 Dez. 6.5 M. Z. Berlin.

$$
\begin{array}{rlrl}
M & =121^{\circ} & 15^{\prime} & 55^{\prime \prime} .87 \\
\pi & =310 & 4 & 9.77 \\
\omega & =234 & 27 & 55.02 \\
\delta & \left.=\begin{array}{rrr}
25 & 36 & 14.75
\end{array}\right\} \text { 1910.0 } & \varphi=4^{\circ} 37^{\circ} 35^{\prime \prime} 68 \\
\mu & =404^{\prime \prime 2} 9239 \\
\log a & =0.6288740
\end{array}
$$

Heliozentrische Äquatorealkoordinaten 19 ro.0.

$$
\begin{aligned}
& x=[9.9996499] \cdot r \cdot \sin \left(165^{\circ} 35^{\prime} 32^{\prime \prime} .05+u\right) \\
& y=[9.9606586] r \cdot \sin (76374.92+u) \\
& z=[9.6 \text { I } 17745] r \cdot \sin (7027 \\
& 7.46+u)
\end{aligned}
$$

\begin{tabular}{|c|c|c|c|c|}
\hline 1907 & $\alpha$ & $\delta$ & $\log r$ & $\log A$ \\
\hline Oct. 20 & $2^{\mathrm{h}}$ I $7^{\mathrm{m}} 12^{\mathrm{s}}$ & $+12^{\circ} \quad 0^{\prime} .5$ & 0.3479 & 0.0936 \\
\hline 22 & $\begin{array}{ll}.15 & 27\end{array}$ & I I 43.4 & & \\
\hline 24 & 1340 & 1126.2 & & \\
\hline 26 & I1 53 & I I 9.1 & & \\
\hline 828 & I0 6 & 1052.2 & $0.35^{15}$ & $0.098 \mathrm{I}$ \\
\hline 30 & 820 & IO 35.4 & & \\
\hline Nov. I & $63^{6}$ & IO I 9.0 & & \\
\hline 3 & 454 & I0 $\quad 2.9$ & & \\
\hline 5 & 314 & 947.2 & $0.355 \mathrm{I}$ & 0.1083 \\
\hline 7 & I 39 & 932.0 & & \\
\hline 9 & $\circ 7$ & +917.4 & & \\
\hline
\end{tabular}

Schlachtensee, 1907 Sept. 30 .

\section{A. Wedemeyer.}

Ephemeris of (518) Halawe.

The following ephemeris is based on the elements given in the Berliner Jahrbuch for I 909 , and gives mean positions I907.0 for Berlin midnight.

Magnitude I 2.7. AR. $\pm \mathrm{I}^{\mathrm{m}}$, Decl. $\pm 5: 5$.

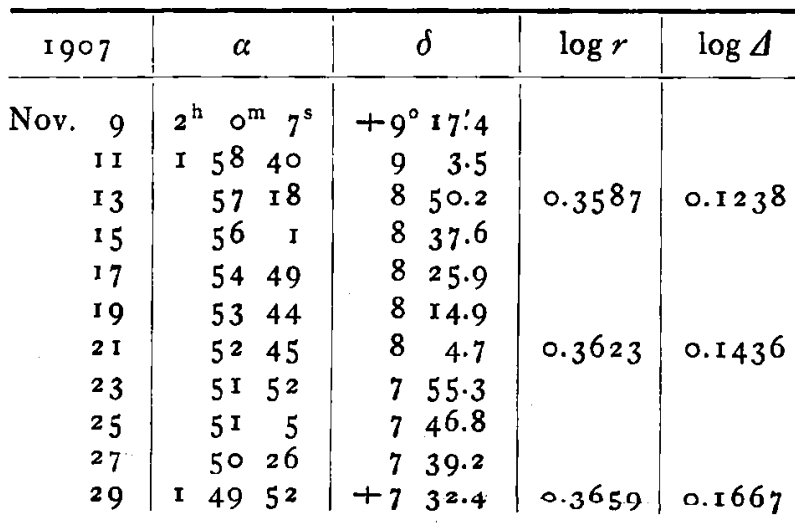

Precession to $1855.0:-2^{m} 4^{6} \cdot 4-14: 6$.

R. S. Dugan.

Princeton University Observatory, I 907 Sept. 28.

\title{
Mitteilung über das Septemberminimum und die Periode von RZ Ophiuchi.
}

Der interessante Algolveränderliche RZ Ophiuchi, ủber dessen Lichtwechsel und Periode noch einige Unsicherheit herrschte, ist von mir am 22., 23, 24., 25., 26., 28, 29. und 30 . September im konstanten Minimallicht beobachtet worden. In den Schätzungen am 20 . September ist das erste Anzeichen einer Helligkeitsabnahme angedeutet und am 2. Oktober fand ich den Stern bereits in voller Maximalgröße am Himmel vor. Der größte Teil des Abstiegs muß somit im Laufe des 21 . September und der Aufstieg im Laufe des x. Oktober erfolgt sein. An diesen beiden Tagen war hier der Hinmel völlig bedeckt, so daß mir leider die beiden wichtigsten Stücke der Kurve entgangen sind. Immerhin läßt sich aus den. Beobachtungen entnehmen, daß ein Minimum I 907 Sept. 26 eingetreten ist, wodurch der Nachweis für die Richtigkeit der zweiten Periode von Blažko (26r.8 Tage) erbracht ist. Der ganze Lichtwechsel umfaßt etwa I 2 Tage, wovon etwa je $1 \frac{1}{2}$ Tage auf die Ab- und Zunahme der Helligkeit entfallen dürften. Während des 9-tägigen Mini. mums bleibt der Stern absolut unveränderlich. Der Iicht. wechsel wird hier offenbar hervorgebracht durch einen Körper, dessen Dimensionen gegenuber dem helleren Stern sehr groß sein müssen, selbst dann, wenn man eine nahezu zentrale Bedeckung annimmt. Dementsprechend ist auch die Existenz eines Nebenminimums bei RZ Ophiuchi sehr wenig wahrscheinlich, und meine zahlreichen Beobachtungen des Sterns geben auch tatsächlich nicht den geringsten Anhaltspunkt für eine Helligkeitsänderung während des 250-tägigen Maximallichtes. Eine ausfuhrliche Mitteilung und Diskussion der hiesigen Schätzungen von RZ Ophiuchi, die I905 Sept. I 8 beginnen, wird an anderer Stelle erfolgen.

\section{K. Graff.}

(471) Papagena. Korrektion der Ephemeride (A. N. 4200): 1907 Okt. $4-5^{\mathrm{m}} \mathrm{I}^{\mathrm{s}}-44: 8$. Gr. 9\%. W. Luther.

Inhalt zu Nr. 4205. M.W. Whitney. Observations of variables. 65. - F. Holetschck. Eine Bemerkung über die Bahnelemente des Kometen I813 I. $67 .-7$. Bauschinger. Numerierung neu entdeckter kleiner Planeten. $69 .-$ F. Bauschinger. Vorläufige Elemente neu entdeckter kleiner Planeten. 71. - E. Tringali. Elementi parabolici della cometa 1907 a. 73. - E. Millosevich. Elementi della cometa 1907 d. 75. - Fr. Schwab. Beobachtungen des Kometen 1907 d. 75. - A. Kopff. Photographische Aufnahmen von kleinen Planeten. 77. - A. Wedemeyer. Ephemeride des Planeten (279) Thule. 77. - R. S. Dugan. Ephemeris of (518) Halawe. 79. - K. Graff. Mitteilung über das Septemberminimun und die Periode von R7 Ophinchi. 79. - W. Luther. (471) Papagena. 79. 